\title{
VANET-based Secure Information Exchange for Smart Charging
}

\author{
Joy Iong-Zong Chen, \\ Department of Communication Engineering, \\ Da-Yeh University, \\ 112 Shan -Jeau, Rd.Da-Tsuen, Chang-Hua, 51505, \\ Taiwan R.O.C
}

\begin{abstract}
Smart inventions have led to smart grids, which have paved the way to smart charging means. This smart charging information about a vehicle needs to be properly maintained in order to use it to exchange information between charging stations and electric vehicles. In this work, we have introduced an efficient methodology for managing and disseminating energy from the charging station to the smart vehicle in an urban area where the number of electric vehicles is high. We have designed and implemented a novel concept involving mobile edge computing in VANET. Moreover, we have also used an effective mechanism for communicating the information on charging with the moving electric vehicles and servers. A local relay scheme is used for reducing redundant overheads, increase delivery efficiency of charging information. This scheme is implemented with delay wait model as the base. The output is recorded by means of simulation environment and based on the observations the proposed work is found to be optimal in maintaining, accessing and disseminating the charging information.
\end{abstract}

Keywords: Smart Charging, Smart Grid, Electric Vehicles, Vehicular Ad-Hoc Network, mobile edge computing

\section{Introduction}

Traffic noise problems along with air pollution issues have been the root cause of several health hazards and environmental deterioration in the past few decades [1]. Though air pollution has been curbed by the introduction of Electric Vehicles, the problem of traffic continues to persist and innovative measures have been introduced in terms of smart parking, IoT-enabled traffic monitoring system etc, to make our means of commute easier [2-3]. Electric vehicles are the chosen mode of commute by many people nowadays because of the environment-friendly implications such low noise, absence of carbon emissions, improved energy efficiency etc. However, there is a demand for the charging aspect of these Electric Vehicles as the number of charging stations and the provision of electricity from the existing grid systems is tested. This might also lead to high impact damage in the already available electric grid system resulting in deterioration of quality in terms of waiting time because of the large number of electric vehicles and limited energy source. In order to address these issues and discrepancies, we propose a novel method to implement the charging services within smart g rids. This method will be able to choose the optimal time as well as the apt charging system (CS) to provide charge to the electric vehicles. It is crucial that a proper channel of communication is established between the CSs and the mobile EVs to process as well as exchange information regarding charging decisions [4], busy situations of the CSs [5], locations of the sources and destination [6] etc.

In the currently prevalent communication framework, charging of EVs take place using systems based on cloud computing. However, there are several security concerns and privacy issues when cloud computing comes into play. Moreover, the cost of communication is also found to be very high in these methodologies [7]. Hence to overcome these difficulties, our methodology guarantees to provide better services with development and advances in technology. The introduction of $5 \mathrm{G}$ in communication has paved way to the use of an innovative network with economic communication expenses, adaptive environment and suitable charging scenario. VANET which is one of the most commonly used method for information dissemination also struggles [8] with many difficulties like fragile link connection, heavy communication overhead, and low adaptively etc. Integrating cellular networks and integrating VANETs, we can gather real-time data [9] to curb these negative effects. However, such a process will require pre-processing and will also introduce heavy loads on the system. In this proposed work, we use an effective strategy to deliver charging information in localities where the number of electric vehicles is high. We have incorporated edge computing servers to execute our criteria in line with VANETS [10]. The first step in our work is to design a framework which serves as a means to establish communication between the charging stations and the electric vehicles [11]. Taking into consideration the realtime traffic in a particular locality, a VANET with an efficient charging information delivery methodology is considered. To improve charging packet delivery ratio and decrease the network overheads, an improved local charging data relaying technique is also used [12].

The novel contribution s of this proposed work is as follows: 
Journal of Electrical Engineering and Automation (EEA) (2020)

Vol. 02/ No. 03

Pages: 141-145

https://www.irojournals.com/iroeea

DOI: https://doi.org/10.36548/jeea.2020.3.006

- Improved Local Relaying Technique: To choose the right next forwarding node, a local relaying scheme is designed such that a waiting time model is used taking into consideration bit error rate and forwarding progress distance.

- Data Delivery: Using a non-deterministic optimization problem, an adaptive system for establishing optimal path of data transmission is constructed with limitation on quality of service. We have introduced a hybrid Ant colony optimization methodology to fend the drawbacks of limited network scalability, high convergence time and rapidly-varying network topologies.

- Communication Framework: Using mobile edge computing, our work ensures smart grid robustness, enhanced scalability, improved privacy and security of EV users, reduction in computational costs and congestions in networks.

\section{Proposed Work}

In this paper we have introduced a model based on quality to ensure that a proper means of communication is established between the source and destination in a particular 2-lane road segment. This methodology will be useful in determining proper charging information delivery and to evaluate the packet delivery ratio. We assign the value of ' 1 ' to the road segment such that ' $d$ ' should satisfy either one of the two conditions of $s>R$ or $s \leq R$ which is the distance between the VANET communication range of the two vehicles. Here $\mathrm{R}$ is used to represent the range of wireless communication. It is also possible to bridge the link between the vehicles by those that are travelling in the opposite direction. This methodology is executed taking into assumption that the vehicles are travelling along Poisson Distribution law, in a two lane road segment. Further, we also take the two opposite directions that the road segment represent to be Westbound and Eastbound. During the operation process, it proves to be hindering some to find the right means of communication in a typical VANET surrounding because of the difficult nature of vehicle mobility.

Westbound

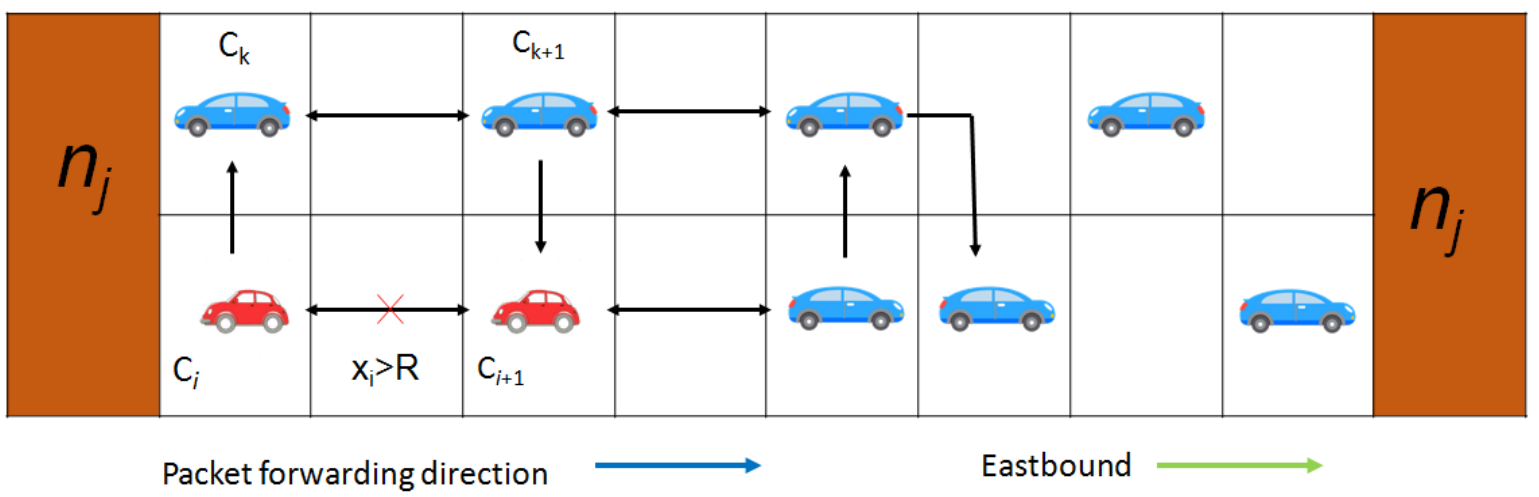

Fig.1. Vehicle Connectivity with double-lanes

To record the characteristics of connectivity we divide the road into clusters of cells as shown in Fig.1. When the distance between two vehicles is represented by $d$ such that its value doesn't go beyond the limit $R$, the connectivity between the two vehicles is guaranteed. On the other hand, was far as cells are considered, the electric vehicles are assumed to be distributed randomly such that the distance between vehicles is within $[0,2 R]$. It is enough that the value is $0.5 \mathrm{R}$, though it is not the necessary criteria for establishing and maintaining connection between 2 EVs. In contrast, it cannot be guaranteed that when two vehicles are within the connectivity range of $\mathrm{R}$, proper connection is stabled even though it is the essential condition to establish connectivity. To overcome this drawback, we fix the cell's size to be $\alpha$ such that it is within the limits 0.5 and 1 . If the length of the road interval is represented by $\alpha . R$, the Probability Mass Function of connection K can be determined as shown in eq (1)

$$
P(K=k)=(\alpha \cdot R \cdot \lambda)^{k} \cdot e^{-k \pi \lambda / k !}
$$

where vehicular spacing density on road segment is represented by $\lambda$. Between two vehicles that are situated adjacent to each other, if the distance exceed R, they are disconnected, along the Eastbound lane. On the other hand, along the westbound land, even if the link is broken, it can be restored using relaying support. Based on these conditions the fixable probability $P_{f}(i)$ can be represented as shown in eq (2). 
Journal of Electrical Engineering and Automation (EEA) (2020)

Vol. 02/ No. 03

Pages: 141-145

https://www.irojournals.com/iroeea

DOI: https://doi.org/10.36548/jeea.2020.3.006

$$
P_{f}(i)=(1-P(K=0))^{\frac{s}{\alpha . R}}
$$

This probability gives a possible chance for repairing the broken connection between two adjacent electric vehicles. If we take into assumption that the number of broken lines in a connection is ' $\mathrm{M}$ ', then the possibility of fixing these links is possible using the moving vehicles' relay functions. The conditional connectivity probability can be represented as:

$$
\begin{gathered}
P_{b \mid M}(M=m)=\sum_{n=1}^{m} P_{f}(l)=\left(1-e^{-\alpha \cdot R \cdot \lambda_{2}}\right)^{\sum_{n=1}^{m} \frac{\left\lfloor x_{b}\right.}{\alpha \cdot R\rfloor}} \\
=\left(1-e^{-\alpha \cdot R \cdot \lambda_{2}}\right)^{\left(\frac{L}{\alpha \cdot R}\right)}
\end{gathered}
$$

Here, $\mathrm{n}$ denotes the vehicle number, $\mathrm{L}$ is the 2-lane length and the vehicle space density is represented by the symbol ' $\lambda$ '. When there are $m$ number of broken links, then the probability mass function of the proposed work can be expressed as:

$$
P_{M}(M=m)=\left(\begin{array}{c}
o-1 \\
m
\end{array}\right) \cdot P_{b}^{m} \cdot\left(1-P_{b}\right)^{N-1-m}
$$

Perfect transmission of information is possible using an intersection-based routing technology tha is recommended by us. This includes an objective function which can be expressed as:

$$
\max _{z} G(z)=\prod P_{d}\left(e_{i j}\right)
$$

Here, the backbone routing path is denoted by the letter ' $z$ ' and is made up of a series of intersections.

\section{Results and Discussion}

In order to execute our proposed work, we built a simulation area of dimensions $5500 \mathrm{~m}$ x $7500 \mathrm{~m}$ holding 86 two-land roads and 31 intersections. Apart from this, we also used 38\% ratio of electric vehicles using discharging or charging requirements with respect to the total number of EVs. The penetration level of the vehicles is fixed within the range of $18 \%$ and $35 \%$. We have made use of an Intelligent Driver Model which incorporates the use of actual location of the vehicles such that their velocity is within the range of 15 to 25 meters per second. The channel capacity of the model used is $7 \mathrm{Mbps}$ and the range of wireless communication network falls within 312 to $420 \mathrm{~m}$. A brief overview of the simulation parameters used are given in the Table 1

TABLE 1. Parameter under consideration in the Proposed Work

\begin{tabular}{|l|c|}
\hline \multicolumn{1}{|c|}{ Parameter } & Value \\
\hline Weight & 0.5 \\
\hline MAC layer protocol & 802.11 \\
\hline Discount factor value (Maximum) & 0.90 \\
\hline Discount factor value (Minimum) & 0.35 \\
\hline Range of Communication & $15 \%$ to 32\% \\
\hline EV penetration & $18 \%$ to 35\% \\
\hline Channel Capacity & 7 Mbps \\
\hline Vehicle Spacing Density & 0.015 \\
\hline Vehicle Velocity & $15-25 \mathrm{~m} / \mathrm{s}$ \\
\hline Mobility Model Used & Intelligent Driver Model \\
\hline Simulation Area & $5500 \mathrm{~m} \times 7500 \mathrm{~m}$ \\
\hline
\end{tabular}


Journal of Electrical Engineering and Automation (EEA) (2020)

Vol. 02/ No. 03

Pages: 141-145

https://www.irojournals.com/iroeea

DOI: https://doi.org/10.36548/jeea.2020.3.006

Fig. 2 represents the probability of connectivity when the road segment has varying cell sizes as well as different ranges of communication. We have considered the length of the road to be $1250 \mathrm{~m}$ for the proposed work. It is observed from the figure that the when the size of the cell is about half that of the cell range.

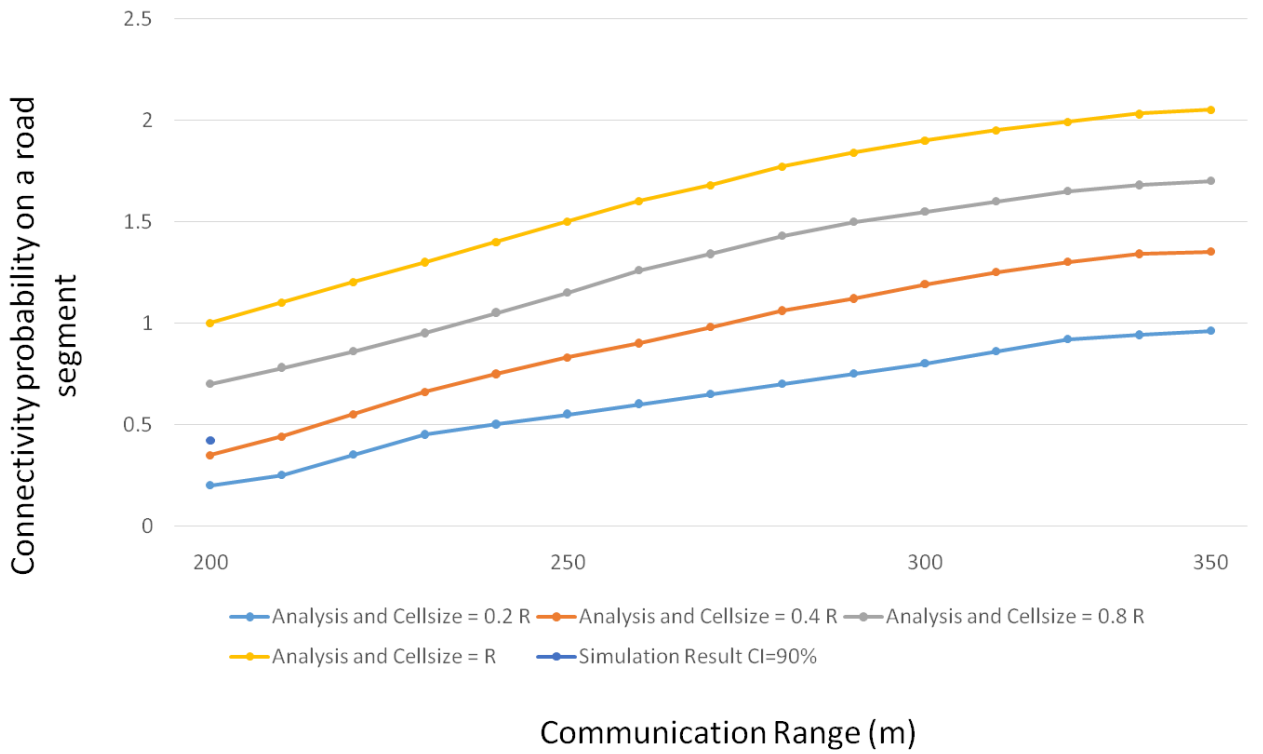

Fig.2. Communication Connectivity based on cell size and range

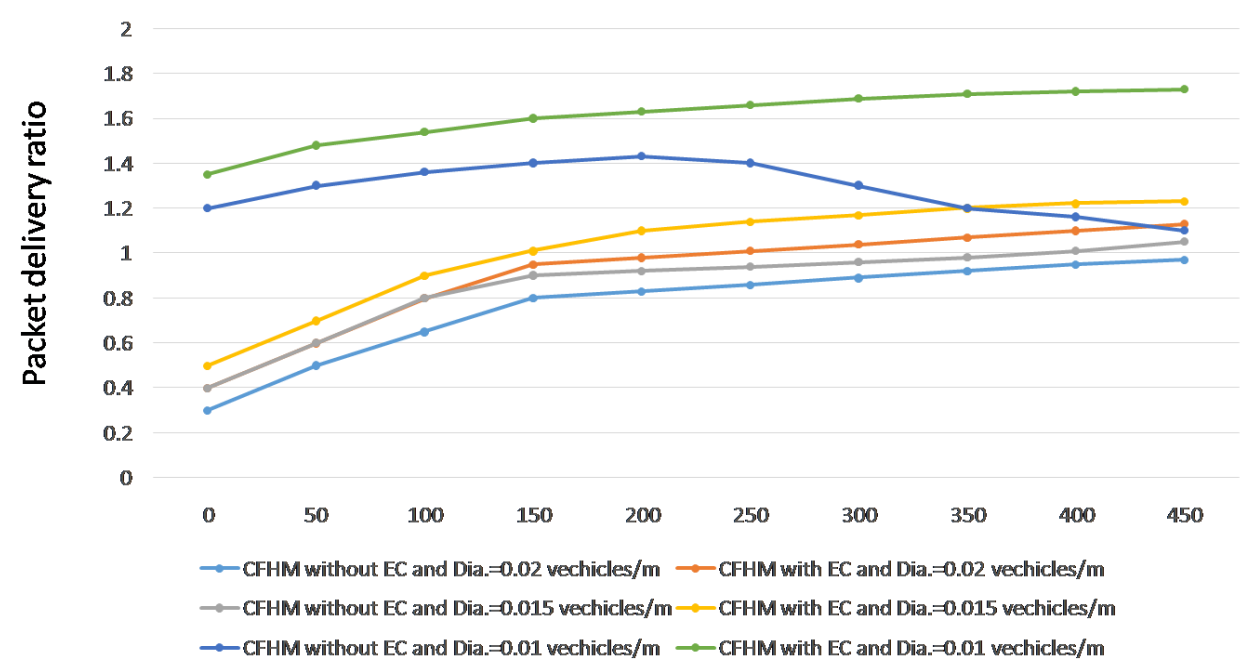

Number of ESs distributed in the urban scenario

Fig.3. Ratio of packet delivery ratio between EV and ES

Using the proposed methodology, with respect to the different edge computing services (ES) the ratio end-to-end packet delivery between mobile electric vehicles and charging stations are taken into account. The results obtained indicate that the proposed work using VANET are more efficient with the edge computing services include MEC functions.

\section{Conclusion}

In this work we have introduced a charging strategy that is used to ensure perfect communication between the vehicles and charging stations. We have used a Q-learning best algorithm for secure transmission of charging details between the moving electric vehicles and edge computing servers. Based on a hybrid framework of communication between the charging station and the electric vehicle, the best route is chosen in order to transfer the charging information between the two parties. To ensure secure and safe transmission, the algorithm is implemented with verification of the node reception. In further work, the proposed technology can be implemented 
Journal of Electrical Engineering and Automation (EEA) (2020)

Vol. 02/ No. 03

Pages: 141-145

https://www.irojournals.com/iroeea

DOI: https://doi.org/10.36548/jeea.2020.3.006

by adding additional features such as automatic smart parking with respect to charging stations. Also, an adaptive mechanism that uses the valules of weights to determine the charging service location can also be implemented as a future progress of the current work.

\section{References}

[1] Bautista, P. B., Cárdenas, L. L., Aguiar, L. U., \& Igartua, M. A. (2019). A traffic-aware electric vehicle charging management system for smart cities. Vehicular Communications, 20, 100188.

[2] Li, G., Li, X., Sun, Q., Boukhatem, L., \& Wu, J. (2020). An Effective MEC Sustained Charging Data Transmission Algorithm in VANET-Based Smart Grids. IEEE Access, 8, 101946-101962.

[3] Azam, F., Priyadarshi, N., Nagar, H., Kumar, S., \& Bhoi, A. K. An Overview of Solar-Powered Electric Vehicle Charging in Vehicular Adhoc Network. Electric Vehicles, 95-102.

[4] Au, M. H., Liu, J. K., Zhang, Z., Susilo, W., Li, J., \& Zhou, J. (2017). Anonymous announcement system (AAS) for electric vehicle in VANETs. The Computer Journal, 60(4), 588-599.

[5] Li, G., Boukhatem, L., Zhao, L., \& Wu, J. (2018, February). Direct vehicle-to-vehicle charging strategy in vehicular ad-hoc networks. In 2018 9th IFIP International Conference on New Technologies, Mobility and Security (NTMS) (pp. 1-5). IEEE.

[6] Cao, Y., Song, H., Kaiwartya, O., Zhou, B., Zhuang, Y., Cao, Y., \& Zhang, X. (2018). Mobile edge computing for big-data-enabled electric vehicle charging. IEEE Communications Magazine, 56(3), 150156.

[7] Khalid, A., Iftikhar, M. S., Almogren, A., Khalid, R., Afzal, M. K., \& Javaid, N. A blockchain based incentive provisioning scheme for traffic event validation and information storage in VANETs. Information Processing \& Management, 58(2), 102464.

[8] Shirley, D. R. A., Ranjani, K., Arunachalam, G., \& Janeera, D. A. (2020). Automatic Distributed Gardening System Using Object Recognition and Visual Servoing. In Inventive Communication and Computational Technologies (pp. 359-369). Springer, Singapore.

[9] Ponmani, D. S. J., Shirley, D. R. A., Aswini, G. V., Sumathi, K., \& Dally, E. C. (2016). A diagnostic study of power switches. Advances in Natural and Applied Sciences, 10(17), 271-275.

[10] Chen, J. I. Z. (2019). 5G technology and advancements in connected living-comprehensive survey. Journal of Electronics, 1(02), 71-79.

[11] Smys, S., \& Ranganathan, G. (2019). Robot assisted sensing control and manufacture in automobile industry. Journal of ISMAC, I(03), 180-187.

[12] Bindhu, V. (2019). Green cloud computing solution for operational cost efficiency and environmental impact reduction. Journal of ISMAC, 1(02), 120-128. 